\title{
Leader
}

\section{Antimicrobial prescribing}

\author{
Michael B Prentice
}

Increasing antimicrobial resistance is an international public health issue addressed by recent reports from the House of Lords ${ }^{1}$ and the Standing Medical Advisory Committee (SMAC). ${ }^{2}$ The spectre hanging over these learned deliberations is a return to the preantibiotic era where patients with common bacterial infections died for want of effective treatment. ${ }^{3}$ The systematic review of (largely circumstantial) published evidence incorporated in the SMAC report suggests that antimicrobial resistance follows antimicrobial use as night follows day. ${ }^{2}$ The challenge is therefore not prevention of the emergence of resistance but rational management of an inevitable evolutionary process ${ }^{4}$ using a population genetics based analysis of epidemiological evidence allied to clinical and laboratory studies. For example, a quantitative relation between changing resistance patterns and antimicrobial use on a national scale $^{56}$ has been established and may suggest ways of rotating availability of different antibiotics nationally in response to surveillance data. ${ }^{4}$ Other mathematical models have suggested that combination, not rotation, is the best option to delay the emergence of resistance. ${ }^{7}$ Further exposure of these models to the real world of surveillance data is required.

Prescribed antimicrobials are not the only selection pressure driving bacterial evolution to antimicrobial resistance. First, in many countries (such as the United Kingdom) only about $50 \%$ of antimicrobial use is in man; $50 \%$ is in animals (mostly used for growth promotion). ${ }^{2}$ Second, antimicrobial use in some countries (particularly developing countries) is not only on prescription ${ }^{8}$ and may be in subtherapeutic regimens with poorly active compounds. ${ }^{9}$

Changes in these areas are matters of national and international governmental policy, ${ }^{12}$ where physicians and microbiologists must seek to inform and influence but have no direct control. Individual prescriptions are, however, completely under the control of individual rational physicians. Eighty per cent of antimicrobials prescribed for human use in the United Kingdom are oral preparations in the community, and $50 \%$ of this community antibiotic use is for respiratory tract infection. ${ }^{2}$ The United Kingdom is a minor player in the world league of antimicrobial consumption: a mere $2 \%$ of the world market, well behind the USA $(50 \%)$ and Japan $(25 \%){ }^{2}$ However, ample data show that antibiotic prescriptions for sore throat, ${ }^{10}$ otitis media, ${ }^{11}$ and lower respiratory tract infection ${ }^{12}$ in United Kingdom general practice are greatly in excess of evidence based clinical indications. Individual agents are being used for inappropriate indications, for example fluoroquinolone prescriptions in the United Kingdom show a winter peak suggesting their use for respiratory tract infections. ${ }^{2}$ Antimicrobial prescribing turns out to have a lot to do with the psychology of the general practitioner-patient relationship. Prescribing behaviour is influenced by the doctor's prior information about a patient, ${ }^{13}$ and by the patient's expectations of the consultation. ${ }^{14} \mathrm{~A}$ cycle of mutual expectation seems to operate where the general practitioner prescribes an antibiotic to avoid the effect of patient expectation manifesting either as a repeat consultation $^{14}$ or a change of practitioner. ${ }^{15}$ Once antibiotic prescription has occurred, representation is in fact more likely. ${ }^{16}$ Rational prescribing in general practice is also hindered by inadequate laboratory data on local epidemiology patterns. ${ }^{17}$

So, is the more impersonal atmosphere in hospital conducive to rational prescribing? In theory, much more surveillance data on prevalent isolates are available and specialist advice from microbiologists based on these data should inform all antibiotic prescriptions through an antibiotic policy, as recommended in the SMAC report. ${ }^{2}$ Infection control procedures within hospital also remain a key part of the process of slowing the emergence of resistant organisms as hospital pathogens and of minimising patient morbidity in the face of endemic resistant organisms. ${ }^{18}$ Although an early landmark success in ending an outbreak of resistant organisms in a high use hospital area involved withdrawal of all antibiotics, ${ }^{19}$ abolishing selective pressure, policy led interventions have since concentrated on replacement of problematic agents by another antimicrobial and the use of cost-effective regimens. ${ }^{18}$ Unfortunately, the efficacy of hospital antibiotic policies in preventing the emergence of resistance is disputed even among microbiologists, ${ }^{20}$ arguably simply shifting the selection pressure towards a different set of resistant organisms. ${ }^{20}{ }^{21}$ Another major drawback cited is an inability to guarantee to a concerned clinician that an individual infected patient does not have an infection resistant to the
Accepted for publication 21 July 1999 
standard empirical regimen ("How can you deny my desperately ill patient life saving wondercillin therapy just to save a few pounds?"). ${ }^{20}$

The first of these criticisms may be answered by guidance from the population genetics analyses referred to above, allowing resistance to be driven in a controlled manner rather than followed expectantly. The second is more difficult to dismiss: the relative costs associated with expensive parenteral antibiotics can be offset by marginal decreases in length of hospital stay. ${ }^{22}$ Uncomfortably, it has also been claimed that even where there is still an additional cost per patient, empirical prescribing of the broadest spectrum agent available for acute septicaemic illness produces enhanced survival at a marginal cost lower than for standard interventions such as antihypertensive treatment or renal dialysis. ${ }^{22}$ This is difficult to reconcile with the extensive data from neutropenic patients showing little difference between various regimens in patient outcome in recovery from sepsis. ${ }^{23}$ However, if a conflict between responsibility to the individual patient (better chance of survival with the broadest spectrum agent) and to the community at large (increased prevalence of resistance to the broad spectrum agent with use, rendering it ineffective in a shorter time) is confirmed in other studies, the risks and benefits of each option may be quantified by the same mathematical tools used to inform vaccination policy. ${ }^{24}$

What can be done to optimise antimicrobial prescribing? The SMAC report recommends an extensive combination of measures including patient education, increased surveillance of resistance patterns, and expert decision systems to guide prescription. ${ }^{2}$ Some of the main recommendations (no prescribing of antibiotics for simple colds and coughs or viral sore throats) illustrate the requirement for extensive education of patients and doctors. Many other measures are already in hand. Unreliable sensitivity test data may be resolved by better controlled methods. ${ }^{25}$ More data on general practitioners' prescribing is now available through electronic PACT (Prescribing Analyses and CosT) data. ${ }^{26}$ The acceptance of standardised evidence based management guidelines from the National Institute of Clinical Excellence ${ }^{27}$ specifying drug, dose, and duration will help both hospital clinicians and general practitioners to adhere to policies. Rapid diagnostic testing may be a logical tool to decrease unnecessary prescribing. ${ }^{2}$ In principle a positive result will inform the choice of appropriate antibiotics: a reliable negative result may reassure both doctor and patient that no antibiotic treatment is required. However, there are few data showing that diagnostic information actually affects prescribing, indeed the value of diagnostic testing in general practice is currently controversial. ${ }^{28}$ Trials of the effect of rapid diagnostic provision on antimicrobial prescribing and increased surveillance of antimicrobial resistance in the hospital and community to provide data for mathematical models are urgently required. More information is also needed on outcome: the adverse consequences of laboratory detected resistance on outcome of infection in the community are poorly documented and need to be quantified for the development of an optimal strategy. ${ }^{4}$ Perhaps the most important factor is a recognition of antimicrobial resistance as a global problem requiring action from governmental level down to each individual prescriber and patient.

1 Select Committee on Science and Technology. Resistance to antibiotics and other antimicrobial agents. London: House of Lords, 1998.

2 Standing Medical Advisory Committee. The path of least resistance. London: Department of Health, 1998.

3 Cohen ML. Epidemiology of drug resistance: implications for a post-antimicrobial era. Science 1992;257:1050-5.

4 Anderson RM. The pandemic of antibiotic resistance. Nature Med 1999;5:147-9.

5 Austin DJ, Kakehashi M, Anderson RM. The transmission dynamics of antibiotic-resistant bacteria: the relationship between resistance in commensal organisms and antibiotic consumption. Proc R Soc Lond B Biol Sci 1997;264:162938 .

6 Austin DJ, Kristinsson KG, Anderson RM. The relationship between the volume of antimicrobial consumption in human communities and the frequency of resistance. Proc Natl Acad Sci USA 1999;96:1152-6.

7 Bonhoeffer S, Lipsitch M, Levin BR. Evaluating treatment protocols to prevent antibiotic resistance. Proc Natl Acad Sci USA 1997;94:12106-11.

8 Okeke IN, Lamikanra A, Edelman R. Socioeconomic and behavioral factors leading to acquired bacterial resistance to antibiotics in developing countries. Emerg Infect Dis 1999;5:18-27.

9 Hart CA, Kariuki S. Antimicrobial resistance in developing countries. BM7 1998;317:647-50.

10 Little P, Williamson I, Warner G, et al. Open randomised trial of prescribing strategies in managing sore throat. BMF 1997;314:722-7.

11 Cates C. An evidence based approach to reducing antibiotic use in children with acute otitis media: controlled before and after study. $B M F$ 1999;318:715-16.

12 Macfarlane J, Lewis SA, Macfarlane R, et al. Contemporary use of antibiotics in 1089 adults presenting with acute lower respiratory tract illness in general practice in the UK: implications for developing management guidelines. Respir Med 1997;91:427-34.

13 Nazareth I, King M. Decision making by general practitioners in diagnosis and management of lower urinary tract symptoms in women. BMF 1993;306:1103-6.

14 Macfarlane J, Holmes W, Macfarlane R, et al. Influence of patients' expectations on antibiotic management of acute ower respiratory tract illness in general practice: questionnaire study. BMF 1997;315:1211-14.

15 Hutchinson JM, Foley RN. Method of physician remuneration and rates of antibiotic prescription. Can Med Assoc 7 1999;160:1013-17.

16 Little P, Gould C, Williamson I, et al. Reattendance and complications in a randomised trial of prescribing strategies for sore throat: the medicalising effect of prescribing antibiotics. BMf 1997;315:350-2.

17 Swann RA, Clark J. Antibiotic policies - relevance to general practitioner prescribing. Family Health Services Authority, Great Britain. If Antimicrob Chemother 1994;33(suppl A): $131-5$

18 Gould IM. A review of the role of antibiotic policies in the control of antibiotic resistance. I Antimicrob Chemother 1999;43:459-65.

19 Price DJ, Sleigh JD. Control of infection due to Klebsiella aerogenes in a neurosurgical unit by withdrawal of all antibiotics. Lancet 1970;ii:1213-15.

20 George R, Scott G, McNulty C, et al. Debate: do antibiotic policies have an effect? $\mathcal{F}$ Hosp Infect 1997;36:85-93.

21 Burke JP. Antibiotic resistance-squeezing the balloon? FAMA 1998;280:1270-1.

22 Leibovici L, Shraga I, Andreassen S. How do you choose antibiotic treatment? BMF 1999;318:1614-18

23 Hughes WT, Armstrong D, Bodey GP, et al. 1997 guidelines for the use of antimicrobial agents in neutropenic patients with unexplained fever. Infectious Diseases Society of America. Clin Infect Dis 1997;25:551-73.

24 Anderson RM, Donnelly CA, Gupta S. Vaccine design, evaluation, and community-based use for antigenically valuation, and community-based use for antigen

25 Wise R. BSAC standardised disc sensitivity testing method. Newsletter of the British Society of Antimicrobial Chemotherapy, 1998 (Summer)

26 Majeed A, Evans N, Head P. What can PACT tell us about prescribing in general practice? BMf 1997;315:1515-19.

27 Rawlins $M$. In pursuit of quality: the National Institute for Clinical Excellence. Lancet 1999;353:1079-82.

28 Kolmos H, Little P. Should general practitioners perform diagnostic tests on patients before prescribing antibiotics? diagnostic tests on patien
BMF 1999;318:799-802 